\title{
Metabolic coupling in pancreatic beta cells: lipolysis revisited
}

\author{
Hindrik Mulder ${ }^{1}$
}

Received: 23 August 2016 / Accepted: 7 September 2016 / Published online: 22 September 2016

(C) Springer-Verlag Berlin Heidelberg 2016

Keywords Adipose triglyceride lipase $\cdot$ Beta cells . Hormone-sensitive lipase $\cdot$ Lipolysis $\cdot$ Metabolic coupling

$\begin{array}{ll}\text { Abbreviations } \\ \text { ATGL } & \text { Adipose triglyceride lipase } \\ \text { GH } & \text { Growth hormone } \\ \text { GLP-1 } & \text { Glucagon-like peptide-1 } \\ \text { HSL } & \text { Hormone-sensitive lipase } \\ \text { PPAR } & \text { Peroxisome proliferator-activated receptor delta } \\ \text { RIP } & \text { Rat insulin promoter }\end{array}$

In this issue of Diabetologia, Marc Prentki and his group [1] add another piece to the puzzle of how lipids are involved in the metabolic control of insulin secretion. While it is generally accepted that increases in blood glucose are transmitted into metabolic changes inside the beta cell, triggering secretion of insulin, the way in which insulin secretion is amplified and sustained is less clear but likely as important,. The work by Attané and colleagues [1] enhances our understanding of the role of lipid metabolism in this context and may shed further light on why insulin secretion fails in type 2 diabetes.

In the article, the authors inactivated adipose triglyceride lipase (ATGL) in pancreatic beta cells [1]. This enzyme is a catalyst for the first committed step in the breakdown of triacylglycerols (Fig. 1) [2]; it hydrolyses triacylglycerols to diacyl-

Hindrik Mulder

hindrik.mulder@med.lu.se

1 Unit of Molecular Metabolism, Lund University Diabetes Centre, Malmö, Sweden glycerols. These are then hydrolysed by the hormone-sensitive lipase (HSL) into monoacylglycerols, which are finally degraded to fatty acids and glycerol [1]. Needless to say, this is a crucial series of metabolic reactions in adipose tissue, allowing our body to access energy in fasting states and during starvation. However, it is generally less appreciated that cells, other than fat cells, may utilise the same reactions, albeit for different purposes.

Marc Prentki, Barbara Corkey and colleagues introduced the concept of lipid signalling as part of the metabolic control of insulin secretion at the end of the 1980s [3]. Their model was inspired by the Randle cycle, in which metabolism of glucose inhibits that of lipids and vice versa. The impact of lipids, either as triacylglycerol or as long-chain acyl-CoA, has underpinned studies of beta cell dysfunction (i.e. lipotoxicity) [4] and beta cell stimulus-secretion coupling (i.e. lipid signalling) [5]. In the context of the latter, the demonstration that insulin secretion occurs independently of activation of the ATP-dependent $\mathrm{K}^{+}$ channel $\left(\mathrm{K}_{\mathrm{ATP}}\right)$ was a seminal finding [6]; it introduced a scenario where lipid signalling fitted snugly. Much of the subsequent work on lipid signalling in beta cells has aimed to tease out the underlying biochemical pathways and molecular mechanisms by which these signals may amplify the secretion of insulin $[7,8]$. This is the general context in which we should place the recent work by Attané et al.

The authors report that male mice (on a normal diet) lacking ATGL exhibit lower fed blood glucose levels and release less insulin, both in vivo and in vitro. In islets, this correlates with lower levels of monoacylglycerol, the endproduct of lipolysis, which is one of several putative lipid signalling molecules that is thought to couple lipid metabolism in beta cells to insulin secretion. Indeed, resupplying monoacylglycerol to knockout mouse islets in vitro restored the impairment of insulin secretion [1].

Based on their findings, the authors conclude that lipolysis in beta cells exerts control over insulin secretion [1]. Exactly 


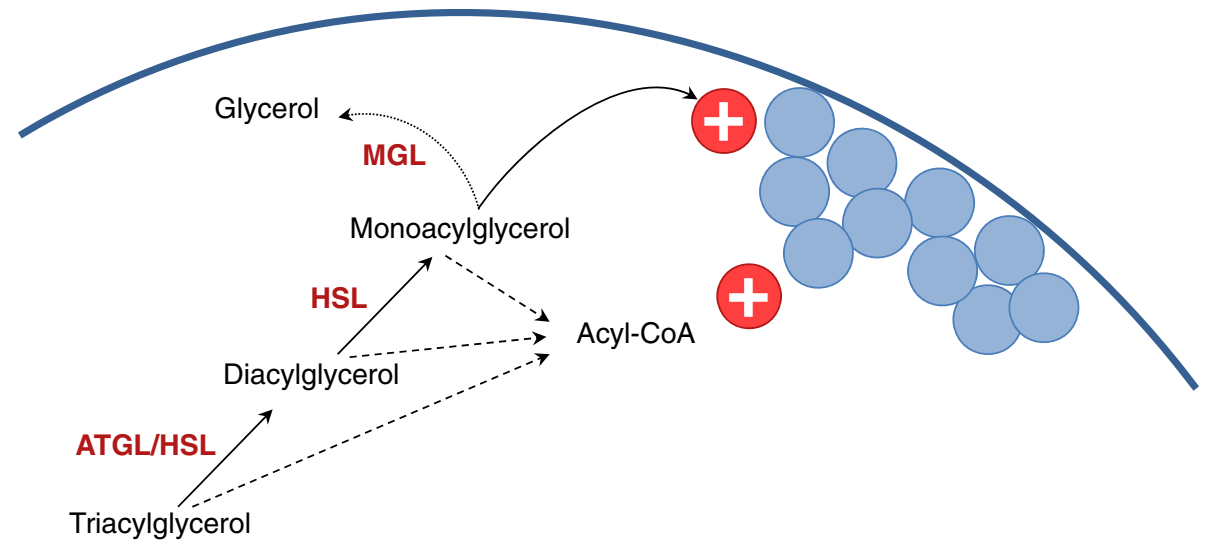

Fig. 1 Lipid signaling in the pancreatic beta cell. Cellular stores of triacylglycerol are hydrolysed by ATGL and HSL to diacylglycerol. In the next rate-limiting step of lipolysis, HSL hydrolyses diacylglycerol to monoacylglycerol. Monoacylglycerol has been proposed to exert a direct stimulatory effect on the exocytotic machinery. Finally, monacylglycerol is degraded to glycerol by monoacylglycerol lipase (MGL) [9]. In each hydrolytic step a fatty acid is liberated. The fatty acid derivatives, acyl-

how this comes about was not shown in the study. However, inferring from previous work, two routes exist: first, monoacylglycerol species, which were reduced in the study by Attané et al, are thought to exert a direct stimulatory effect on the exocytotic machinery (a similar effect has previously been attributed to long-chain acyl-CoA [3], which is also formed in lipolysis; Fig. 1). This is corroborated by previous findings by Marc Prentki's group, showing that blockade of an enzyme that hydrolyses monoacylglycerol, a membranebound monoacylglycerol lipase ( $\alpha / \beta$-hydrolase domain-6), results in increased insulin secretion in mice, and that monoacylglycerol binds to Munc13, a protein involved in exocytosis [9]. Second, Prentki and associates have proposed the existence of a glycerolipid/NEFA cycle in beta cells [10]. While this cycle is futile in terms of energy production, the input of monoacylglycerol may drive a lipogenic arm of the cycle, leading to production of lipid species, which may serve to amplify insulin secretion by mechanisms remaining to be shown.

While the findings so far pertaining to the metabolic control of insulin secretion are of great interest, they are also not surprising. Mice with whole body ATGL knockout also exhibit impaired insulin secretion [11], as do mice with beta cell-specific knockout of HSL [12]. Moreover, another group recently reported that beta cell-specific ATGL knockout mice exhibit impaired insulin release [13], although there are several discrepancies in the phenotypes of the two ATGL knockout lines, as discussed below.

Instead, a surprising finding from the study by Attané et al demonstrates that the development of high fat diet-induced obesity was slowed down in male ATGL knockout mice. This retardation was accompanied by better insulin tolerance and a trend towards lower fasting blood glucose. ATGL
CoAs, have also previously been suggested to act as coupling signals in insulin secretion [2]. Blue circles represent insulin-containing granules; red circles with a plus sign illustrate a stimulatory signal for insulin release; the blue semi-circle represents the plasma membrane. Continuous arrows show committed formation of acylglycerol moieties, while dashed arrows indicate the repeated formation of acyl-CoA in each step and the dotted arrow shows the formation of glycerol

knockout mice of both sexes also released less insulin during an oral glucose tolerance test, as did their isolated islets in vitro. In light of this, the authors suggest that insulin secretion was curtailed in ATGL knockout mice, protecting them from diet-induced obesity and, potentially, also from the development of hyperglycaemia and ultimately diabetes [1]. Indeed, normally a drive towards hyperglycaemia and hyperinsulinaemia is observed in animal models receiving an energy surplus. This is typically explained by the development of insulin resistance, which is established very quickly in, for example, mice of the C57/BL/6J background (with or without the nicotinamide nucleotide transhydrogenase [NNT] deletion) given a high fat diet, for which increased insulin secretion occurs as a compensatory mechanism [14]. Instead, however, the authors argue that the excess fuel results in obesity and diabetes owing to increased secretion of insulin [1]. This may be understood in light of the anabolic effects of insulin, leading, for example, to the accumulation of lipids in peripheral tissues, while inhibiting break down of lipids in adipose tissue. Based on this reasoning, the authors suggest that interventions that actually lower the secretion of insulin may be of potential therapeutic use in obesity and diabetes [1]. Clearly, this is at odds with the established therapeutic paradigm of increasing insulin secretion to lower blood glucose when type 2 diabetes evolves. Current therapy employs sulfonylureas, meglitinides and, more recently, incretin-based modalities, such as glucagon-like peptide-1 (GLP-1) analogues and/or inhibitors of incretin degradation (dipeptidyl peptidase-4 [DPP-IV] inhibitors), to achieve this therapeutic goal. Ultimately, insulin therapy is used in type 2 diabetes when blood glucose can no longer be controlled by the aforementioned drugs. Although there is some evidence in the literature [1], very little work has been reported in humans to 
support this controversial suggestion. Having stated this, those of us who see patients can recount numerous examples of patients gaining weight upon insulin treatment, leading to further development of insulin resistance, factors which do not benefit the patient in the long term. However, this is normally not seen with treatment by GLP-1 analogues, which, in addition to an insulinotropic effect, promote weight loss.

The authors report that energy expenditure is increased in ATGL knockout mice during the night when the animals are feeding [1]. This is reflected by increases in lipolysis, HSL activation and in fat oxidation in adipose tissue. These processes may partly explain why reduced secretion of insulin is associated with slower weight gain in ATGL knockout mice.

The fascinating findings by Attané et al raise a number of important questions; (1) in their model, the genetic ablation of ATGL in the beta cell is certainly the primary event but how does it result in enhanced insulin sensitivity? Clearly, fine mapping of peripheral glucose uptake, hepatic glucose production etc. with clamp studies would help to clarify this; (2) Is it possible that manipulation of beta cell lipid metabolism specifically exerts this effect or does it also apply to the metabolism of other fuels? As far as I know, interfering with beta cell function does not generally lead to enhanced insulin sensitivity, perhaps in an attempt to compensate for the reduction in insulin secretion; (3) What is the signal mediating this effect? A panel of plasma lipids was unchanged in ATGL knockout mice on a high fat diet, therefore, plasma lipids do not appear to play a role. Is it insulin itself or are other processes at play in the regulation of insulin secretion, perhaps neural or humoral? Clearly, if such a signal were to be identified, it would hold great promise in becoming a novel therapeutic modality in type 2 diabetes and obesity; (4) What underlies the sex difference in the impact of beta cell knockout of ATGL on whole body metabolism?; (5) Does this imply that lipid signalling in beta cells is one of many redundant processes that serves to amplify insulin secretion?

Another outstanding question is the discrepancy with a previous study of ATGL knockout mice [13]. While both groups report an abrogation of insulin secretion [1, 13], Tang et al demonstrated that their lines of ATGL knockout mice (they used both a constitutive rat insulin promoter [RIP]-Cre and a RIP-CreER promoter to create a beta cell null mutation) were glucose intolerant, with insulin tolerance being unaffected. In contrast to the data reported by Attané et al, there also appeared to be beta cell compensation in these mice, which was evidenced by increased beta cell mass. The mice generated by Tang et al also exhibited impaired mitochondrial metabolism in pancreatic beta cells, resulting in diminished ATP production and insulin release. They explain their findings by a lack of production of peroxisome proliferatoractivated receptor delta (PPAR $\delta$ ) ligands, attributed to reduced lipolysis of triacylglycerols and a subsequent reduction in the production of lipid species serving as PPAR $\delta$ ligands [13].
With the cost of generating transgenic mice declining, we are now fortunate enough to see examples of multiple groups using this technology to address important research questions, whilst independently targeting the same gene. Thus, how is it possible that two groups, with well-controlled experiments, report such different results? It has become increasingly clear that individual lines of transgenic mice used in these types of experiments may have design features with varying metabolic implications. For instance, it was recently shown that some frequently used driver transgenic mouse lines express a growth hormone $(\mathrm{GH})$ mini gene in pancreatic beta cells [15]; this has an impact on insulin content and beta cell mass. Attané et al performed the appropriate control experiments on their Mip-Cre-ERT mice, which express the GH mini gene [1]. Tang et al [13] state that their mice were acquired from Jackson Laboratory and thus can be assumed, but not confirmed, to also express the $\mathrm{GH}$ mini gene. If nothing else, these circumstances again underscore the importance of reproducing results and using different approaches to establish biological facts. Confirmation of important results is an undervalued aspect of today's science, where there is a constant hunt for exciting and novel findings. The field is looking forward to further unravelling the role of lipids in beta cell stimulussecretion coupling.

Duality of interests The author declares that there is no duality of interest associated with this manuscript.

Contribution statement The author is the sole contributor to this paper.

\section{References}

1. Attané C, Peyot M-L, Lussier R et al (2016) A beta cell ATGLlipolysis/adipose tissue axis controls energy homeostasis and body weight via insulin secretion in mice. Diabetologia. doi:10.1007 /s00125-016-4105-2

2. Zimmermann R, Strauss JG, Haemmerle G et al (2004) Fat mobilization in adipose tissue is promoted by adipose triglyceride lipase. Science 306:1383-1386

3. Corkey BE, Glennon MC, Chen KS, Deeney JT, Matschinsky FM, Prentki M (1989) A role for malonyl-CoA in glucose-stimulated insulin secretion from clonal pancreatic beta-cells. J Biol Chem 264:21608-21612

4. Unger RH (1995) Lipotoxicity in the pathogenesis of obesitydependent NIDDM. Genetic and clinical implications. Diabetes 44:863-870

5. Nolan CJ, Prentki M (2008) The islet beta-cell: fuel responsive and vulnerable. Trends Endocrinol Metab 19:285-291

6. Gembal M, Detimary P, Gilon P, Gao ZY, Henquin JC (1993) Mechanisms by which glucose can control insulin release independently from its action on adenosine triphosphate-sensitive $\mathrm{K}+$ channels in mouse B cells. J Clin Investig 91:871-880 
7. Cnop M, Foufelle F, Velloso LA (2012) Endoplasmic reticulum stress, obesity and diabetes. Trends Mol Med 18:59-68

8. Biden TJ, Boslem E, Chu KY, Sue N (2014) Lipotoxic endoplasmic reticulum stress, beta cell failure, and type 2 diabetes mellitus. Trends Endocrinol Metab 25:389-398

9. Zhao S, Mugabo Y, Iglesias J et al (2014) alpha/betaHydrolase domain-6-accessible monoacylglycerol controls glucose-stimulated insulin secretion. Cell Metab 19:9931007

10. Prentki M, Madiraju SR (2008) Glycerolipid metabolism and signaling in health and disease. Endocr Rev 29:647-676

11. Peyot ML, Guay C, Latour MG et al (2009) Adipose triglyceride lipase is implicated in fuel- and non-fuel-stimulated insulin secretion. J Biol Chem 284:16848-16859
12. Fex M, Haemmerle G, Wierup N et al (2009) A beta cell-specific knockout of hormone-sensitive lipase in mice results in hyperglycaemia and disruption of exocytosis. Diabetologia 52: 271-280

13. Tang T, Abbott MJ, Ahmadian M, Lopes AB, Wang Y, Sul HS (2013) Desnutrin/ATGL activates PPARdelta to promote mitochondrial function for insulin secretion in islet beta cells. Cell Metab 18:883-895

14. Fex M, Dekker Nitert M, Wierup N, Ling C, Sundler F, Mulder H (2007) Enhanced mitochondrial metabolism may account for the adaptation to insulin resistance in islets from high fat diet-fed C57BL/6J mice. Diabetologia 50:74-83

15. Brouwers B, de Faudeur G, Osipovich AB et al (2014) Impaired islet function in commonly used transgenic mouse lines due to human growth hormone minigene expression. Cell Metab 20:979-990 